\title{
Effect of increasing doses of beta agonists on spirometric parameters, exercise capacity, and quality of life in patients with chronic airflow limitation
}

Roman Jaeschke, Gordon H Guyatt, Andrew Willan, Deborah Cook, Sandi Harper, Joanne Morris, Helen Ramsdale, Roger Haddon, Michael Newhouse

\begin{abstract}
Background - A study was undertaken to determine the impact of different doses of inhaled terbutaline on peak flow rates, spirometric parameters, functional exercise capacity, and quality of life in patients with chronic airflow limitation. Methods - A double blind, randomised, placebo controlled, multiple crossover trial was conducted with treatment periods of one week. Patients with a clinical diagnosis of chronic airflow limitation and $\mathrm{FEV}_{1}$ below $70 \%$ predicted after administration of bronchodilator were recruited from secondary care respiratory practices, and the effect of 500 , 1000 , and $1500 \mu \mathrm{g}$ inhaled terbutaline four times daily on spirometric parameters $\left(F_{1}\right.$, FVC), maximum inspiratory pressures, six minute walking distance, and health-related quality of life (Chronic Respiratory Disease Questionnaire, Quality of Well Being, Standard Gamble) was measured.
\end{abstract}

Results - Twenty five patients completed the trial. Peak flow rates and FEV showed statistically significant but clinically trivial improvement on the higher drug doses. Results of maximum inspiratory pressure measurements, walk test distance, and quality of life measures showed minimal differences on the different dosages, and none of the differences approached conventional statistical significance.

Conclusions - Regular use of $\beta$ agonists in doses higher than two puffs four times a day is very unlikely to provide additional functional or symptomatic benefit to patients with chronic airflow limitation.

(Thorax 1994;49:479-484)

Mnter University Canada

Astra Pharma Inc. J Morris

Reprint requests to: Dr R Jaeschke, St Joseph's Hospital, Fontbonne Bldg, 50 Chariton Av East Hamilton, Ontario, Canada L8N 4A6.

Received 17 May 1993 Returned to authors 25 August 1993 Revised version received 9 November 1993 Accepted for publication 24 January 1994 - may be of benefit. Physicians frequently prescribe multiple drug regimens, especially in more severely affected patients, and there is considerable potential for side effects and non-compliance. The choice of a clinically includions have shown that many drus pium bromide 45 and glucocorticosteroids "optimal" dose regimen is therefore of major importance.

Inhaled $\beta$ agonist therapy not only improves airflow limitation in patients with chronic airflow limitation, but also improves exercise capacity, functional capacity, and healthrelated quality of life. ${ }^{19}$ On the basis of these findings, clinicians frequently prescribe inhaled $\beta$ agonists as the first line treatment for patients with poorly reversible chronic airflow limitation.

In the past few years controversy has arisen as to the optimal dose of inhaled $\beta$ agonists. Available data generally suggest that, in patients with poorly reversible airway obstruction, doses higher than those used conventionally in clinical practice result in increased bronchodilation. ${ }^{10-17}$ Similar results have been reported in patients with asthma. ${ }^{18-20}$ One interpretation of these results is that clinicians should be prescribing larger doses of $\beta$ agonists to their patients. In the recent guidelines for the assessment and management of chronic airflow limitation issued by the Canadian Thoracic Society the authors conclude that "although two puffs from a metered dose inhaler four times daily is a common first step, greater benefit may be achieved with four or six puffs at each administration."

Clinicians might, however, be sceptical about this conclusion. Firstly, the largest bronchodilator response comes from the initial dose of $\beta$ agonist and the subsequent incremental response, although statistically significant, may not translate into clinically important gains in functional status. ${ }^{11}$ Secondly, the conclusions of these studies were based on the acute responses to $\beta$ agonists over a period of a few hours. Assuming that long term functional benefit parallels acute increase in $\mathrm{FEV}_{1}$ following larger doses of $\beta$ agonist may be inappropriate. Thirdly, the finding of a large, consistent increment in $\mathrm{FEV}_{1}$ with increasing doses of bronchodilator has not been universal. Finally, patients may not take large doses of inhaled bronchodilators on a regular basis. ${ }^{1}$

In an attempt to determine the optimal dose of $\beta$ agonists in chronic airflow limitation, we undertook a study in which we examined the relative impact of different doses of inhaled terbutaline taken over periods of one week on peak flow rates, spirometric parameters, functional exercise capacity, and quality of life. 


\section{Methods \\ PATIENTS}

We have described the initial process of patient recruitment in detail elsewhere. ${ }^{11}$ In short, patients were recruited from a registry of more than 1000 patients with chronic airflow limitation (defined as best $\mathrm{FEV}_{1}$ less than $70 \%$ predicted and an $\mathrm{FEV}_{1} / \mathrm{FVC}$ ratio of less than 0.7 on at least $80 \%$ of all measurements during the previous two years). The inclusion criteria, confirmed on the first visit, were as follows: (1) FEV, less than $70 \%$ of predicted value and FEV 1 /FVC ratio less than 0.7 following eight puffs of inhaled bronchodilator $(800 \mu \mathrm{g}$ salbutamol or $2000 \mu \mathrm{g}$ terbutaline); and (2) age 40 years or older.

Patients were excluded by the presence of any of the following: (1) inability to cooperate with procedures because of factors such as limited ability to communicate in English, or cognitive impairment; (2) clinical instability as indicated by hospitalisation in the two months before entry or change in respiratory medications in the month before study entry; (3) known hypersensitivity to sympathomimetic amines or aerosol propellants; (4) history of cardiac tachyarrhythmias requiring treatment; (5) inability to identify activities associated with shortness of breath; and (6) inability to tolerate withdrawal from bronchodilators used before the trial (inhaled or systemic steroids were left at a constant dose).

\section{STUDY DESIGN}

Before the trial each patient attended three visits at weekly intervals. During this run in period all regular bronchodilators were withdrawn, inhaled or systemic steroids were kept at a constant dose, and the patients were given $1.5 \mathrm{mg}$ inhaled terbutaline using a metered dose inhaler four times a day. On two visits each patient underwent a dose titration study of inhaled terbutaline. In this process we identified the "optimal dose" of inhaled terbutaline which was conservatively defined as the dose that led to an $\mathrm{FEV}$, at least $50 \mathrm{ml}$ greater than any other dose.

The metered dose inhaler technique was reviewed with each patient at the initial run in visit. If patients showed suboptimal technique they were instructed in the appropriate method and practiced with a placebo puffer until they had mastered the technique. Three patients in whom optimal technique was not achieved used a valved holding chamber (Aerochamber) for administration of bronchodilator throughout the study.

Each patient then participated in two "triplets" of treatment periods. Each triplet consisted of one week taking terbutaline $0.5 \mathrm{mg}$ (two puffs) four times daily and additional placebo, one week taking terbutaline $1 \mathrm{mg}$ (four puffs) four times daily and additional placebo, and one week taking terbutaline either $1.5 \mathrm{mg}$ (six puffs) four times daily or their optimal dose, whichever was higher. Blinding was achieved by giving patients three inhalers. During the low dose periods only the first inhaler contained active medication; dur- ing the medium dose periods the first two inhalers contained active medication; and during the high dose periods all three inhalers contained active medication. The order of the doses within each triplet was determined by random allocation.

\section{OUTCOME MEASURES}

Patients measured their peak flow rates each day 20 minutes after the use of an evening dose of the medication using a peak flow meter (HealthScan Inc, New Jersey, USA) and recorded the best of two measurements. All other outcomes were obtained at the "end of period" study visit which, for each patient, occurred at the same time of day. A research assistant assigned to each patient administered the outcome measures at each study visit.

Forced expiratory volume in one second $\left(\mathrm{FEV}_{1}\right)$ and forced vital capacity (FVC) were measured with a Collins water spirometer with a 420 microprocessor (Warren E Collins, Braintree, Massachusetts, USA); the $\mathrm{FEV}_{1}$ from the best of two forced expired manoeuvres from total lung capacity to residual volume were recorded. Respiratory muscle strength was tested at functional residual capacity (FRC) by measuring maximum inspiratory pressures with the use of a manual manometer calibrated in $\mathrm{cm} \mathrm{H}_{2} \mathrm{O}$, with sealed mouthpiece and nose clips; the best of three efforts was recorded. The six minute walking test was carried out in a quiet, closed corridor, $30 \mathrm{~m}$ in length, using previously described methods including standardised encouragement. ${ }^{22}$

A disease-specific health-related quality of life (HRQL) questionnaire which has proved responsive - that is, it can detect clinically important changes even if these changes are small - and valid - that is, it is really measuring disease-specific HRQL - in previous work, ${ }^{23}$ the Chronic Respiratory Disease Questionnaire (CRQ), was administered at the end of each treatment period. The CRQ measures physical and emotional function. Physical function assessment included asking patients to quantitate their dyspnoea during three to five activities that are frequently performed and important in their day to day lives, and four items relating to fatigue and energy level. Questions regarding emotional function included frustration, depression, anxiety, fear, and panic with dyspnoea. Patients were asked to rate their function on each item using an appropriate seven point scale - for example, extremely short of breath; very short of breath; ... not at all short of breath. The results were expressed as the score per question in each dimension. The average minimal important difference is 0.5 point per question - that is, subjects whose scores increase or decrease by 0.5 or more generally experience clinically important improvement or deterioration. ${ }^{24}$

The Quality of Well Being instrument (QWB) was used as a generic utility HRQL questionnaire. The QWB represents a preference or value which members of the community associate with the particular combination of functions (mobility, physical 
activity, and social activity) and symptoms (such as cough, wheezing, or shortness of breath). The score varies from 0 (death) to 1 (full health). Although the QWB score was developed as a general HRQL instrument, it has proved valid and responsive as an outcome measure for evaluating interventions for patients with chronic airflow limitation. ${ }^{25}$

To further measure the value which patients put on their overall health a second utility measure - the Standard Gamble - was used in which patients are asked to make a single rating which takes into account all aspects of their HRQL. ${ }^{26}$ Specifically, patients are asked to choose between their own health state and a gamble in which they may die immediately or achieve full health for the remainder of their lives. Patient utility is determined by the highest probability of death they are willing to accept and still choose the gamble. The smallest difference detected by the instrument, as we used it, is 0.05 .

\section{COMPLIANCE}

Throughout the run in and double blind phases the canisters were weighed before and after study periods and compliance calculated as a percentage of the expected use. In addition, patients were asked how often they missed a scheduled dose and how often they had to take extra puffs.

\section{SAFETY}

Patients were asked not to use any extra bronchodilators or extra puffs of terbutaline beyond those prescribed. They were instructed, however, that if they absolutely had to use extra puffs, one puff from each puffer could be used. Patients were asked to contact a member of the study team who was available 24 hours a day, seven days a week, if they felt the need to use extra puffs. Physicians thus contacted made a clinical judgement as to whether a true exacerbation had occurred or whether the increasing symptoms were consistent with a change in the study medication or with the patient's usual variability in symptoms.

\section{TREMOR AND OTHER SIDE EFFECTS}

The presence and severity of tremor was evaluated by two methods during each study visit 15 minutes after the dose of bronchodilator was administered. Firstly, using the "drawing method" 27 we asked the patient to draw a line between two parallel sine waves $2 \mathrm{~mm}$ apart and the number of errors was counted. Secondly, the research assistant used a scale from 0 (tremor absent) to 6 (severe tremor present when arm flexed) to assess the degree of tremor present with the patient's arms extended in front of her/him, and then flexed with elbows facing out and fingers facing each other.
STATISTICAL ANALYSIS

A repeated measures analysis of variance appropriate for multiple crossover designs with factors of drug dose (low, medium, or high), triplet (first or second), and the interaction between the two was used. This analysis was repeated for all the continuous outcome variables. We looked for order effects within triplets by comparing treatment effects in instances when high dose preceded low dose with treatment effects when low dose preceded high dose. Differences in proportions were analysed using $\chi^{2}$ and tests for marginal homogeneity. Because we undertook multiple comparisons a $\mathrm{p}$ value of 0.01 was used for statistical significance.

\section{Results}

\section{PATIENT RECRUITMENT}

A total of 1091 patients were identified who, according to chart review, met the spirometric criteria and were potentially eligible for the study; 237 of these patients had died, 135 could not be located, and a further 324 were judged by their physicians to be unsuitable for the study (most commonly "too sick" or "too anxious"). Of the remaining 395 patients 284 were contacted of whom 50 agreed to participate and started the run in period. Fifteen of these 50 did not complete the run-in period, seven because they were ineligible $\left(\mathrm{FEV}_{1}\right.$ $>70 \%$ predicted in two patients, inability to identify activities associated with shortness of breath in four, and language barrier in one), and eight withdrew (six experienced exacerbations of their symptoms with withdrawal of their regular bronchodilators other than $\beta$ agonist, one could not tolerate substitution of regular salbutamol by terbutaline, and one did not like to take puffers). Of 35 patients who completed the run in period eight declined further participation (five could not continue further without ipratropium, two were judged to be too symptomatic to continue weekly visits, and one found puffers too irritating). Of the 27 patients who began the two triplets two did not complete the trial; one decided he could not continue without ipratropium while the other was withdrawn by his respiratory physician because of a progressive decline in functional status. Neither of these two patients completed the first treatment triplet. The trial was interrupted in two patients; one developed an infective exacerbation and the other had severe chest wall pain following a prolonged coughing spell. Both restarted the study after returning to their usual state of health, and data from the treatment triplet in which the illness occurred were excluded from the analysis.

\section{PATIENT CHARACTERISTICS}

The characteristics of the 25 patients who completed the trial are summarised in table 1 . 
Table 1 Mean (SD) baseline characteristics of participating patients

\begin{tabular}{|c|c|}
\hline Number of patients $(M: F)$ & $25(18: 7)$ \\
\hline \multicolumn{2}{|l|}{ Primary diagnosis } \\
\hline Chronic bronchitis & 11 \\
\hline Emphysema & 2 \\
\hline Asthma & 3 \\
\hline Chronic airflow limitation & 9 \\
\hline Age (range) (years) & $68(5 \cdot 6)(55-77)$ \\
\hline Smoking history (pack years) & $40(24)(1-110)$ \\
\hline $\mathrm{FEV}_{1}$ (range) (1) & $0.94(0.32)(0.30-1.46)$ \\
\hline $\mathrm{FEV}_{1}$ (range) (\% pred) & $36(12)(14-55)$ \\
\hline FVC (range) (1) & $2.12(0.56)(1.12-3.13)$ \\
\hline FVC (range) (\% pred) & $57(15)(32-93)$ \\
\hline $\mathrm{FEV}_{1} / \mathrm{FVC}$ (range) & $0.45(0.13)(0.24-0.69)$ \\
\hline \multicolumn{2}{|l|}{ Medications used before study } \\
\hline Beta agonist (single drug) & $25(5)$ \\
\hline Inhaled steroid & 18 \\
\hline Ipratropium bromide & 8 \\
\hline Oral steroid & 6 \\
\hline Theophylline & 4 \\
\hline
\end{tabular}

$\mathrm{FEV}_{1}=$ forced expiratory volume in one second; $\mathrm{FVC}=$ forced vital capacity.

\section{COMPLIANCE}

The results of the run in period during which the optimal dose of terbutaline was established for each patient resulted in high dose being six puffs in 17 patients and eight puffs in the remaining eight. The weight of canisters expressed as a mean (SE) percentage of expected was $97 \cdot 8(2 \cdot 6) \%$ for the treatment periods on low dose, $98 \cdot 4(2 \cdot 3) \%$ for the periods on four puffs four times daily, and $96.9(2 \cdot 0) \%$ for the treatment periods on high dose terbutaline. No evidence of differential compliance was found with different puffers or different dosages. Patients reported missing doses of the drug after $20 \%, 22 \%$, and $28 \%$ of the treatment periods on low, medium, and high dose of terbutaline respectively $(p>0 \cdot 1)$. Use of the extra doses was reported after $26 \%$ of treatment periods on low dose, and $28 \%$ treatment periods on both medium and high dose $(\mathrm{p}>0 \cdot 5)$.

\section{PRIMARY OUTCOMES}

Statistically significant dose effects were found for daily peak flows $(p<0.01)$ and for $\mathrm{FEV}_{1} 20$ minutes after bronchodilator administration $(p<0.005)$. No other dose effects reached statistical significance. In none of the analyses did we find evidence of clinically important order effects which would suggest a decrement in apparent response to high dose when it is given before the low dose. Neither the triplet effect nor the triplet by dose interaction was significant for any of the variables.

The results of all outcome measures obtained on the low dose of terbutaline are presented in table 2 which also shows the differences between these values and the values obtained on each of the higher doses, together with the $95 \%$ confidence intervals around the differences. The peak flow measurements showed a weak dose-response relation with only marginal differences between the three doses. The $\mathrm{FEV}_{1}$ was $50 \mathrm{ml}$ higher on both the medium and high doses than on the low dose. The upper boundary of the confidence interval for the six minute walking test and the HRQL measures excluded a clinically important difference between the higher and lower doses.
TREMOR

The average numbers of errors made while drawing a sine wave were $1.25,1.30$, and 1.18 for the low, medium, and high doses respectively (ANOVA for dose effect $p>0.5$ ). The mean severity of tremor as judged by the research assistant on a six point scale was 0.76 for low dose, 0.86 for medium dose, and 0.92 for high dose (ANOVA $\mathrm{p}>0 \cdot 5$ ).

\section{Discussion}

Our results indicate that in elderly symptomatic patients with chronic airflow limitation with an element of fixed airflow obstruction there is little or no benefit in increasing the dose of regular inhaled $\beta$ agonist beyond two puffs four times a day.

The strengths of our study include random allocation of treatment periods, effective double blinding, intensive study of each patient, and comprehensive measurement of outcomes. Critics might question whether the study had adequate power to detect small but clinically important treatment effects; however, even in the variables that showed statistically significant effects of dose the upper boundaries of the $95 \%$ confidence interval (indicating the largest dose effect compatible with the data) were unimpressive: $91 / \mathrm{min}$ for the peak flow rate and $80 \mathrm{ml}$ for the $\mathrm{FEV}_{1} \cdot{ }^{28} 29$ The walk test results are compatible with an effect of higher doses of $\beta$ agonists of no greater than 13 metres, considerably less than our estimate of the minimally important difference of 30-40 metres.

The same is true for HRQL: the upper confidence limit for each domain of our disease-specific HRQL measure (the CRQ) was less than 0.2 per question, less than half of what we have previously established as change representing a minimal important difference. ${ }^{24}$ In fact, the mean score differences on all domains of this instrument are slightly higher on low dose. The QWB scale, which takes into account broader aspects of health status than the $C R Q$, also showed slightly higher scores on low dose with upper limits of $95 \%$ CI excluding clinically relevant benefit of higher doses.

It is unlikely that our failure to detect effects on HRQL is due to inadequacy of all the measurement instruments. In a double blind randomised trial with a smaller number of subjects we were able to show clinically important and statistically significant effects of theophylline and salbutamol $v$ placebo on each domain of the CRQ. ${ }^{130}$ This strongly suggests that the instrument is able to detect drug effects on HRQL if they exist.

We therefore believe that our results are consistent with previous findings which suggest that large doses of $\beta$ agonists can result in acute improvement in $\mathrm{FEV}_{1}$ but fail to show clear or consistent improvement in functional or HRQL measures. ${ }^{1012-17}$ Could our negative result be a function of the $\beta$ agonist we chose, or the fact that our starting dose was already too large? There are no data to suggest that the biological action of terbutaline is any different from other drugs of this class. Indeed, study 
Table 2 Mean (SD) results of outcome measures on low dose terbutaline ( $0.5 \mathrm{mg}$ four times daily) and mean (95\% confidence intervals) changes in these outcomes on higher doses (1 mg four times daily, $1.5 \mathrm{mg}$ or more four times daily) in comparison with the low dose

\begin{tabular}{lccl}
\hline Outcome & $0.5 \mathrm{mg}$ four times daily & $1 \mathrm{mg}$ four times daily & $1.5 \mathrm{mg}$ or more four times daily \\
\hline FEV 1 (1) & $1.08(0.31)$ & $0.05(0.02$ to 0.08$)$ & $0.05(0.01$ to 0.08$)$ \\
FVC (1) & $2.47(0.59)$ & $0.06(0.0003$ to 0.11$)$ & $0.03(-0.03$ to 0.09$)$ \\
Six minute walking (metres) & $444(81)$ & $4(-4$ to 13$)$ & $-3(-12$ to 6$)$ \\
CRQ dyspnoea* & $4.2(1 \cdot 1)$ & $-0.04(-0.2$ to 0.1$)$ & $-0.1(-0.3$ to 0.1$)$ \\
CRQ fatigue & $4.7(1.4)$ & $-0.03(-0.2$ to 0.1$)$ & $-0.04(-0.3$ to 0.2$)$ \\
CRQ emotional & $5.5(1 \cdot 1)$ & $0(-0.1$ to 0.1$)$ & $-0.01(-0.2$ to 0.2$)$ \\
CRQ mastery & $5.9(1.1)$ & $-0.03(-0.2$ to 0.1$)$ & $-0.05(-0.2$ to 0.1$)$ \\
QWB & $0.75(0.11)$ & $-0.04(-0.08$ to 0.0002$)$ & $-0.02(-0.05$ to 0.02$)$ \\
Standard Gamble & $0.77(0.21)$ & $0.02(-0.01$ to 0.06$)$ & $-0.02(-0.08$ to 0.04$)$ \\
\hline
\end{tabular}

$\mathrm{FEV}_{1}=$ forced expiratory volume in one second; FVC = forced vital capacity; $\mathrm{CRQ}=$ Chronic Respiratory Disease Questionnaire; QWB $=$ Quality of Well Being.

* Score per question.

results have consistently suggested that $200 \mu \mathrm{g}$ salbutamol has comparable bronchodilator effects to $500 \mu \mathrm{g}$ terbutaline. ${ }^{31-33}$ The possibility that freon propellants used in pressurised aerosols $^{34}$ did not allow manifestation of the full benefit of the high dose is unlikely, as we observed a clear dose effect on $\mathrm{FEV}_{1}$ and peak expiratory flow. Finally, the lowest dose we used $(2 \mathrm{mg} /$ day) is consistent both with standard clinical practice and with the doses of $\beta$ agonists used in previous studies indicating the efficacy of these agents.

Treatment periods of one week were used in our study. Although it is possible that longer treatment periods would yield different results, the rapid action of $\beta$ agonists makes it likely that their clinically relevant benefits would become apparent in the first week of treatment. Indeed, in our previous study we documented substantial effects on HRQL within two weeks of changes in treatment. ${ }^{1}$ Furthermore, with longer periods on high doses one could expect that possible tachyphylaxis (if this phenomenon occurs) ${ }^{1217}$ would decrease rather than increase the effect. Indeed, there is now evidence to suggest that regular long term use of bronchodilators may be deleterious in both patients with asthma ${ }^{35}$ and perhaps also those with chronic airflow limitation. ${ }^{36}$ Mechanisms postulated to explain the deleterious effect of regular inhaled $\beta$ agonists in asthma include their effect on stimulating bronchial secretions, ${ }^{37}$ reduced airway calibre due to mucosal vasodilation, and downregulation of $\beta$ receptors. ${ }^{12}$ These mechanisms may also apply to non-asthmatic patients with chronic airflow limitation.

Our study does not exclude the possibility that there are subpopulations of patients with chronic airflow limitation, or idiosyncratic individuals, who achieve important benefit by large doses of $\beta$ agonists. These patients might include those with extremely variable disease or disease that is difficult to control, and such patients may have been differentially excluded from our study. Given that our results suggest that most patients with chronic airflow limitation will not benefit, and that placebo effects, the natural history of chronic airflow limitation (which is characterised by spontaneous remissions and exacerbations), and patient and physician expectations all favour false positive labelling of drug responders, correctly identifying the occasional patient who benefits may be difficult. We have previously suggested a methodology, $N$ of 1 randomised trials, which could aid physicians in determining true drug responders $^{38-40}$ and have demonstrated the application of the methodology to patients with chronic airflow limitation. ${ }^{41}$

In summary, our study indicates that in symptomatic, stable, elderly patients with an element of fixed airway obstruction a dose of inhaled $\beta$ agonist of more than two puffs taken four times a day is not associated with a clinically important improvement in functional exercise capacity or HRQL after one week of treatment. Given the possibility that regular long term use of $\beta$ agonists may even be deleterious, there is currently little justification for administering them in doses of more than two puffs four times a day.

Drs Guyatt and Cook are career scientists of the Ontario Ministry of Health. Drs Cook and Jaeschke are Research Scholars of the St Joseph's Health Care Foundation, Hamilton, Ontario. This study was supported in part by the Ontario Ministry of Health and Astra Pharma Inc. The medications Ministry of Health and Astra Pharma
were provided by Astra Pharma Inc.

1 Guyatt GH, Townsend M, Pugsley SO, Keller JL, Short $\mathrm{HD}$, Taylor DW, et al. Bronchodilators in chronic air low limitation. Am Rev Respir Dis 1987;135:1069-74.

2 Mahler DA, Matthay RA, Snyder PE, Wells CK, Loke J. Sustained-release theophylline reduces dyspnea in nonreversible obstructive airway disease. Am Rev Respir Dis 1985;131:22-5.

3 Murciano D, Auclair M, Pariente R, Aubier M. A randomized, controlled trial of theophylline in patients with severe chronic obstructive pulmonary disease. $N$ Engl $f$ Med 1989;320:1521-5.

4 Connolly CK, Chan NS. Salbutamol and ipratropium in partially reversible airways obstruction. $\mathrm{Br} \mathcal{F}$ Dis Chest 1987;81:55-61.

5 Brown IG, Chan CS, Kelly CA, Dent AG, Zimmerman PV. Assessment of the clinical usefulness of nebulized ipratropium bromide in patients with chronic airflow limitation. Thorax 1984;39:272-6.

6 Harding SM, Freedman S. A comparison of oral and inhaled steroids in patients with chronic airways obstruction: features determining response. Thorax 1978;33: 214-8.

7 Mitchell DM, Rehahn M, Gilden P, Dimond AH, Collins JV. Effects of prednisolone in chronic airflow limitation. Lancet 1984;ii:193-6.

8 Postma DS, Steenhvis EJ, van der Weele Th, Sluiter HJ. Severe chronic airflow obstruction: can corticosteroids
slow down progression? Eur $\mathcal{F}$ Respir Dis 1985;67:56-4.

9 Taylor DR, Buick B, Kinney C, Lowry RC, McDevitt DG. The efficacy of orally administered theophylline, inhaled salbutamol, and a combination of the two as a chronic salbutamol, and a combination of the two as a chronic therapy in the management of chronic bronchitis with
reversible airflow obstruction. Am Rev Respir Dis reversible airflow

10 Jenkins SC, Heaton RW, Fulton TJ, Moxham J. Comparison of domiciliary nebulized salbutamol and salbutamol from a metered-dose inhaler in stable chronic airflow limitation. Chest 1987;91:804-7.

11 Jaeschke R, Guyatt GH, Cook D, Morris J, Willan A, McIlroy W, et al. The effect of increasing doses of beta agonists on airflow in patients with chronic airflow limitation. Respir Med 1993;87:433-8.

12 Georgopoulos D, Wong D, Anthonisen NR. Tolerance to beta-agonists in patients with chronic obstructive pulmonary disease. Chest 1990;97:280-4.

13 Corris PA, Neville E, Nariman S, Gibson GJ. Doseresponse study of inhaled salbutamol powder in chronic response study of inhaled salbutamol pow

14 Assouf BK, Hodson ME. High dose salbutamol in chronic airflow obstruction: comparison of nebulizer with Rotaairfow obstruction: comparison

15 Vathenen AS, Britton JR, Ebden P, Cookson JB, Wharrad 
$\mathrm{HJ}$, Tattersfield AE. High dose inhaled albuterol in severe chronic airflow limitation. Am Rev Respir Dis 1989;138:850-5.

16 Jenkins SC, Moxham J. High dose salbutamol in chronic bronchitis: comparison of $400 \mu \mathrm{g}, 1 \mathrm{mg}$ and $2 \mathrm{mg}$ and bronchitis: comparison of $400 \mu \mathrm{g}, 1 \mathrm{mg}$ and $2 \mathrm{mg}$ and
placebo delivered by Rotahaler. $\mathrm{Br} f \mathrm{f}$ Dis Chest placebo delivere

17 Lipworth BJ, Clark RA, Dhillon DP, McDevitt DG. Comparison of the effects of prolonged treatment with low and high doses of inhaled terbutaline on beta-adrenoceptor responsiveness in patients with chronic obstructive pulmonary disease. Am Rev Respir Dis 1990;142:338-42.

18 Mestitz H, Copland JM, McDonald CF. Comparison of outpatient nebulized vs metered dose inhaler terbutaline in chronic airflow obstruction. Chest 1989;96:1237-40.

19 Prior JG, Cochrane GM Assessment of optimum dose of inhaled terbutaline in patients with chronic asthma: the use of simple, cumulative dose-response curves. $\mathrm{Br} \mathcal{F} \mathrm{Dis}$ Chest 1982;76:266-8.

20 Chaieb J, Belcher N, Rees PJ. Maximum achievable bronchodilatation in asthma. Respir Med 1989;83:497-502.

21 Canadian Thoracic Society Workshop Group. Guidelines for the assessment and management of chronic obstructive pulmonary disease. Can Med Assoc 7 1992;147:420-8.

22 Guyatt GH, Pugsley SO, Sullivan MJ, Thompson PJ, Berman LB, Jones $\mathrm{N}$, et al. Effect of encouragement on walking test performance. Thorax 1984;39:818-22. 23 Guyatt G, Berman L, Townsend M, Pugsley SO, trials in chronic lung disease. Thorax 1987;42:773-8.

24 Jaeschke R, Singer J, Guyatt G. Measurement of health status: ascertaining the minimal clinically important difference. Controlled Clin Trials 1989;10:407-15.

25 Kaplan RM, Atkins CJ, Timms R. Validity of a quality of well-being scale as an outcome measure in chronic well-being scale as an outcome measure in chronic
obstructive pulmonary disease. $\mathcal{f}$ Chronic Dis 1984;37: 85-95.

26 Torrance GW. Measurement of health state utilities for economic appraisal. F Health Econom 1986;5:1-30.

27 Thiringer G, Svedmyr N. Evaluation of skeletal muscle tremor due to bronchodilator agents. Scand $\mathcal{F}$ Respir Dis 1975;56:93-102

28 Tweedale PM, Merchant S, Leslie M, Alexander F, McHardy GJR. Short term variability in $\mathrm{FEV}_{1}$ : relation to pretest activity, level of $\mathrm{FEV}_{1}$, and smoking habits. Thorax 1984;39:928-32.
29 Tweedale PM, Alexander F, McHardy GJR. Short term variability in $\mathrm{FEV}_{1}$ and bronchodilator responsiveness in patients with obstructive ventilatory defects. Thorax patients with o

30 Jaeschke R, Guyatt GH, Singer J, Keller J, Newhouse MT. Mechanism of bronchodilator effect in chronic airflow Mechanism of bronchodilator effect in ch

31 Munzenberger PJ, Papaioanou HA, Massoud N. A clinical comparison of terbutaline with albuterol administered by metered-dose inhaler. Ann Allergy 1989;62:107-10

32 Simonsson BG, Stiksa J, Strom B. Double-blind trial with increasing doses of salbutamol and terbutaline aerosols in patients with reversible airways obstruction. Acta Med Scand 1972;192:371-6.

33 Freedman BJ. Trial of terbutaline aerosol in the treatment of asthma and a comparison of its effects with those of a salbutamol aerosol. Br $\mathcal{A}$ Dis Chest 1972;66:222-9.

34 Editorial. Fluorocarbon aerosol propellants. Lancet 1975;i: 1073-4.

35 Sears MR, Taylor DR, Print CG, Lake DC, Li Q, Flannery $\mathrm{EM}$, et al. Regular inhaled beta-agonist treatment in bronchial asthma. Lancet 1990;336:1391-6.

36 Van Schayck CP, Dompeling E, Van Herwaarden CLA, Folgering H, Verbeek ALM, Van der Hoogen HJM, et al. Bronchodilator treatment in moderate asthma or chronic bronchitis: continuous or on demand? A randomized controlled study. BMF 1991;303:1426-31.

37 Williams IP, Phipps RJ, Wright NL, Pack RJ, Richardson DS. Sympathomimetic agonists stimulate mucus secretion into human bronchi. Thorax 1981;36:231.

38 Guyatt GH, Sackett DL, Taylor DW, Chong J, Roberts R, Pugsley SO. Determining optimal therapy - randomized trials in individual patients. N Engl f Med 1986;314:88992.

39 Guyatt GH, Keller JL, Jaeschke R, Rosenbloom D, Adachi JD, Newhouse MT. Clinical usefulness of $N$ of 1 randomized control trials: three year experience. Ann Intern mized control trials:

40 Guyatt GH, Sackett DL, Adachi JD, Roberts RS, Chong J, Rosenbloom D, et al. A clinician's guide for conducting randomized trials in individual patients. Can Med Assoc $\mathcal{F}$ 1988;139:497-503.

41 Patel A, Jaeschke R, Guyatt G, Newhouse MT, Keller J. Clinical usefulness of $\mathrm{N}$ of 1 RCTs in patients with chronic airflow limitation. $A m$ Rev Respir Dis 1991;14:962-4 